\title{
Prognostic Value of Tumor Infiltrating Lymphocytes in Locally Advanced HER2 Enriched Breast Cancer
}

\author{
Mostafa M Saber'1, Heba M El Zawahry ${ }^{2}$, Amany M Hilal², Amany AAbou-Bakr³, \\ Alfred E Namour ${ }^{2}$, Mona M Saber ${ }^{4 *}$
}

\begin{abstract}
Purpose: We aim to study the association between stromal tumor infiltrating lymphocytes (TILs) level and disease free survival (DFS) in a group of ER and PR negative, HER2+ locally advanced breast cancer patients who underwent curative intent surgery. Methods: This is a retrospective cohort study including 66 locally advanced hormone receptornegative; HER2+ breast cancer patients presented between 2013 and 2015 at NCI-Cairo, Egypt. Enrolled patients had at least clinically T3 and/or node positive disease either clinically or radiologically. Metastatic workup included CT and bone scans or PET-CT. Patients with hormone receptor positive, HER2 negative, inadequate paraffin block and who lost follow up before or immediately after curative surgery were excluded. Patients were followed from breast surgery till relapse date for a minimum of 36 months. TILs and CD8 antigen were assessed on paraffin-embedded blocks using immunohistochemistry. Results: Patients with a median age of 52 years presented with clinical T3 stage (53\%) and N1 stage (61\%). Modified radical mastectomy was performed in 79\%. Only $41 \%$ received neoadjuvant chemotherapy and $56 \%$ received trastuzumab. TILs were 50, 17 and 33\% for absent, intermediate and extensive groups and CD8+ lymphocytes were present in $80 \%$ of cases. At the end of follow-up period, 23 patients $(35 \%)$ were found to have disease recurrence either loco-regional (22\%) or distant (78\%). TILs were 14, 4 and 5\% for absent, intermediate and extensive respectively; while CD8+ lymphocytes were absent in $6 \%$ and present $(\geq 1 \%)$ in $17 \%$. Higher DFS was recorded for patients with extensive TILs level only who received trastuzumab. Conclusion: High TILs is good prognosis in HER2 enriched breast cancer provided that patients received HER2 directed therapy. Moreover, CD8+ lymphocytes are highly representative and maybe used as an alternative for TILs. We recommend considering TILs and specifically CD8+ as one of the risk factors that predict prognosis of HER2+ breast cancer.
\end{abstract}

Keywords: Breast cancer- HER2+- Trastuzumab- Tumor infiltrating lymphocytes- CD8

Asian Pac J Cancer Prev, 23 (2), 553-560

\section{Introduction}

One of the most important goals of cancer research is identification of robust prognostic and predictive markers. In breast cancer, these biomarkers may play an important role to estimate accurately the risk of relapse which allows for optimization of different therapeutic options. Recently, the interplay between immune system and cancer cells has been highlighted. Several studies investigated the interconnection between tumor infiltrating lymphocytes and tumor cell progression, as well as, predicting response to various anti-cancer therapies (Adams et al., 2014).

Clinical outcome does not only depend on the amount but also the phenotype of the infiltrate. Type $1 \mathrm{~T}$-cells are associated with favorable prognosis; CD8+ cytotoxic T-cells (CTL) are important for tumor destruction, CD4+ T-helper 1 (Th1) cells cause activation of antigen presenting cells and secretion of various cytokines which facilitates antigen presentation. On the contrary, type 2 CD4+ T-helper cells (Th2), including Forkhead box P3 (FOXP3) CD4+ regulatory T-cells, inhibit CTL function, and may promote an anti-inflammatory immune response that could amplify tumor growth. In order to facilitate TILs measurement; The International TILs Working Group has developed a standardized evaluation of breast cancer TILs that can be easily applied in clinical practice (Salgado et al., 2015).

Several studies have highlighted the importance of TILs as a prognostic biomarker in non-metastatic breast cancer before starting any line of therapy. Regarding the prognostic effect of cytotoxic CD8+ T cells, the percentage of its presence is strongly associated with prolonged survival outcome and good response to chemotherapy (Liu et al., 2012; Seo et al., 2013).

In 2010, Denkert et al. identified the prognostic value of TILs as a biomarker in breast cancer (Denkert et al.,

${ }^{1}$ Medical Oncology, Faculty of Medicine, Port Said University, Egypt. ${ }^{2}$ Medical Oncology, National Cancer Institute, Cairo University, Egypt. ${ }^{3}$ Pathology, National Cancer Institute, Cairo University, Egypt. ${ }^{4}$ Department of Pharmacology and Toxicology, Faculty of Pharmacy, Cairo University, Egypt. *For Correspondence: mona.magdy@pharma.cu.edu.eg 
2010). It was the first study to evaluate TILs using the protocol of the International TIL working group. Since then, many researchers have focused on the association between presence of TILs and clinical outcome in various breast cancer subtypes. The relevant correlation has been shown among triple negative subtype, followed by the HER2 enriched breast cancer.

To this end, we aim to investigate the association between the level of stromal tumor infiltrating lymphocytes (TILs) and DFS (after a follow-up period of 3 years) in a group of ER andPR negative, HER-2 positive locally advanced breast cancer patients who received curative intent surgery at national cancer institute (NCI), Cairo University from 2013-2015. Moreover, to study the association between the levels of TILs at time of presentation with other prognostic criteria in the same group will be studied.

\section{Materials and Methods}

\section{Ethical approval}

The study protocol was approved by the Institutional Review Board (IRB) of the national cancer institute (NCI). All patients received the NCI standard-of-care therapy. The study was conducted in accordance with the ethical principles stated in the Declaration of Helsinki and the guidelines of Good Clinical Practice (GCP). Patient information was dealt with adequate confidentiality. Results and other study material did not include items that would reveal patients' identities.

\section{Study Design}

This is a retrospective cohort study of patients with locally advanced hormone receptor negative/ HER2 positive breast cancer who presented to medical oncology department of National cancer institute, Cairo University; and diagnosed between 2013 and 2015.

Follow up period of 3 years from date of curative surgery and data was collected from patients' files. Correlation of the clinical outcome with TILs and CD8 status was assessed by IHC on tumor paraffin-embedded blocks. Patients were recruited in the study based on the pathology results, therapy received and surgical intervention. The patients were subjected to full history taking and thorough clinical examination. Base-line metastatic work up including CT chest, abdomen and pelvis as well as bone scan to exclude distant metastasis was performed. Regular follow-up visit for each patient was done according to her treatment schedule, then they were followed-up every 3 months.

\section{Data Collection and follow-up}

A case report form (CRF) was constructed and the data of each patient were recorded including the age, the date of diagnosis of $\mathrm{BC}$, the date and type of surgery, the histo-pathological criteria including: histological subtype of the breast tumor, grade, size, number of positive axillary lymph nodes, clinical and pathological TNM stage and the IHC status of stromal TILs and CD8 percentage. The details of the neoadjuvant and adjuvant therapy were recorded. The date and site(s) of relapse-if present-, the follow up dates, the date of last follow up visit, the status at last follow up and the date of death were all recorded in the CRF. BMI is defined as the body mass divided by the square of the body height, $\left(\mathrm{kg} / \mathrm{m}^{2}\right)$.

Laboratory and radiological investigations were requested upon symptom or clinical suspicion of recurrence (local, contralateral or distant). Once recurrence was documented, follow up period for the study was ended and disease free survival is calculated.

Patients who did not relapse were followed up every 3-6 months for a minimum of 3 years period with regular clinical assessment.

\section{Immunohistochemistry}

Tissue sections were prepared from samples of patients before receiving chemo- or radiotherapy. Biopsy specimens where used for patients who received neoadjuvant chemotherapy. Hematoxylin and Eosin stained slides were reviewed for adequacy of the tissue material, histological type (Tan et al., 2020), histological grade (modified Bloom and Richardson grading system (ELSTON and ELLIS, 1991) presence of carcinoma in situ, lymphovascular invasion and axillary lymph node status. Basic biomarkers estrogen receptor (ER), progesterone receptor (PR), HER2 status, and Ki-67 proliferation index were retrieved. Immunohistochemical assessment for CD8 (SP57), Rabbit monoclonal antibodies (Roche) was performed on routinely processed, formalinfixed, paraffin-embedded tissues. Unstained sections were prepared at 4 microns thickness and mounted on positive charged slides. Immunostaining was done for all cases using BenchMark XT (Ventana) autostainer and the assessment of immunostaining was performed using Olympus light microscope (CX31).

\section{Statistical analysis}

All statistical analysis was performed using IBM SPSS advanced statistics, version 24.0 (SPSS Inc., Chicago, IL, USA). For the examination of qualitative variables Chi-square-Fisher's exact- test was used. Receiver Operating Characteristics (ROC) curve was done to estimate the best cut off point. Sensitivity and specificity was then calculated with their $95 \%$ confidence interval. Kaplan-Meier curves were used for survival analysis after which log rank test was performed to compare between the survival curves. Cox regression model was used for multivariate analysis with calculating hazard ratio and its $95 \%$ confidence interval. Bonferonni corrections of $p$ value was implemented. A p-value $<0.05$ was considered statistically significant and all tests were two tailed. Graphs were performed using Prism software program (GraphPad Prism software, version 7, CA, USA) and analysis of data was performed using GraphPad INSTAT (version 7).

\section{Results}

\section{Patient Characteristics}

Between 2013 and 2015, 90 cases were collected that fulfilled the inclusion criteria. However, paraffin blocks for only 66 of them were recovered.

All 66 patients were females. The median age 
Table 1. Clinico-Pathological Characteristics of the Study Group

\begin{tabular}{|c|c|c|}
\hline Characteristic & Number of patients & Percent \\
\hline \multicolumn{3}{|l|}{ Age } \\
\hline$\leq 65$ years & 62 & 94 \\
\hline$>65$ years & 4 & 6 \\
\hline Pregnancy & 3 & 5 \\
\hline Lactation & 8 & 12 \\
\hline Oral contraceptive pills intake & 23 & 35 \\
\hline \multicolumn{3}{|l|}{ Menopause } \\
\hline Pre & 35 & 53 \\
\hline Post & 31 & 47 \\
\hline Family History & 16 & 24 \\
\hline \multicolumn{3}{|l|}{ BMI } \\
\hline$<30$ & 19 & 29 \\
\hline$\geq 30$ & 47 & 71 \\
\hline \multicolumn{3}{|l|}{ Clinical $(\mathrm{T})$ stage } \\
\hline II & 12 & 18 \\
\hline III & 35 & 53 \\
\hline IV & 19 & 29 \\
\hline \multicolumn{3}{|l|}{ Clinical (N) stage } \\
\hline 0 & 17 & 26 \\
\hline 1 & 40 & 60 \\
\hline 2 & 9 & 14 \\
\hline \multicolumn{3}{|l|}{ TNM stage } \\
\hline IIB & 27 & 41 \\
\hline IIIA & 20 & 30 \\
\hline IIIB & 19 & 29 \\
\hline \multicolumn{3}{|l|}{ Side affected } \\
\hline Right & 33 & 50 \\
\hline Left & 33 & 50 \\
\hline \multicolumn{3}{|l|}{ Tumor site } \\
\hline Central & 16 & 24 \\
\hline Multicentric & 4 & 6 \\
\hline LOQ & 5 & 8 \\
\hline LIQ & 2 & 3 \\
\hline UOQ & 39 & 59 \\
\hline UIQ & 10 & 15 \\
\hline \multicolumn{3}{|l|}{ Surgery } \\
\hline MRM & 52 & 79 \\
\hline CBS & 14 & 21 \\
\hline Neoadjuvant chemotherapy & 27 & 41 \\
\hline Adjuvant chemotherapy & 42 & 64 \\
\hline Adjuvant radiotherapy & 66 & 100 \\
\hline Received Trastuzumab & 37 & 56 \\
\hline \multicolumn{3}{|l|}{ Grade } \\
\hline II & 55 & 83 \\
\hline III & 11 & 17 \\
\hline \multicolumn{3}{|l|}{ Pathological (T) stage* } \\
\hline 0 & 6 & 9 \\
\hline 1 & 4 & 6 \\
\hline 2 & 35 & 53 \\
\hline 3 & 15 & 22 \\
\hline 4 & 7 & 10 \\
\hline
\end{tabular}

Table 1. Continued

\begin{tabular}{lcc}
\hline Characteristic & Number of patients & Percent \\
\hline Pathological (N) stage* & 20 & 30 \\
0 & 17 & 26 \\
1 & 20 & 30 \\
2 & 9 & 13 \\
3 & 17 & 26 \\
LVI & 7 & 26 \\
pCR* & & \\
Incomplete pCR*: & 12 & 44 \\
Mild & 8 & 29 \\
Moderate & & \\
TILs & 33 & 50 \\
Absent & 11 & 17 \\
Intermediate & 22 & 33 \\
Extensive & & \\
CD8+ & 13 & 20 \\
Absent & 53 & 80 \\
Present & &
\end{tabular}

at presentation was 52 years (range 28-71). Clinico-pathological characteristics of the study group are shown in table. Pathological response assessment was estimated using residual cancer burden (RCB) MD Anderson criteria (Symmans et al., 2007). Notably, $44 \%(n=12)$ of the patients who achieved less than pCR received only anthracyclines in neoadjuvant setting. Characteristics of the studied population is shown in a flow chart in Figure 1.

\section{Disease recurrence and characteristics of the relapse}

At the last visit with a median follow up period of 36 months (range 1-70), 35\% ( $\mathrm{n}=23)$ had evidence of relapse. The median time of relapse as calculated from date of curative surgery till date of relapse was 21 (range $1-61)$ months, with $13 \%(n=3)$ relapsing during the first year of follow up.

Analysis of relapsed cases showed that $22 \%(n=5)$ were local recurrence; and $78 \%$ of patient had systemic recurrences. Lung was the commonest site of metastases $(n=8)$. Among the relapsed case, $52 \%(n=12)$ did not receive trastuzumab at any time during the course of therapy.

\section{Tumor infiltrating lymphocytes and CD8+ assessment}

Measuring levels of stromal TILs on paraffin block was done following the international TILs Working Group standard guide for evaluating TILs in clinical practice, that classify stromal TILs into absent $(0-10 \%)$, intermediate $(>10-40 \%)$ and extensive ( $>40-90 \%)$. Figure 2 shows representative images of TILs and CD8 after immunostaining. In this cohort, examination of TILs showed a mean value of $28.3 \%$ (range 1-90), median 15 and mode value of 2 . Results were $50 \%(\mathrm{n}=33), 17 \%$ $(\mathrm{n}=11), 33 \%(\mathrm{n}=22)$ for absent, intermediate and extensive respectively. Out of the extensive subgroup, 9 patients had $>60 \%$ lymphocyte infiltration, which is defined as lymphocyte-predominant breast cancer (LPBC). 


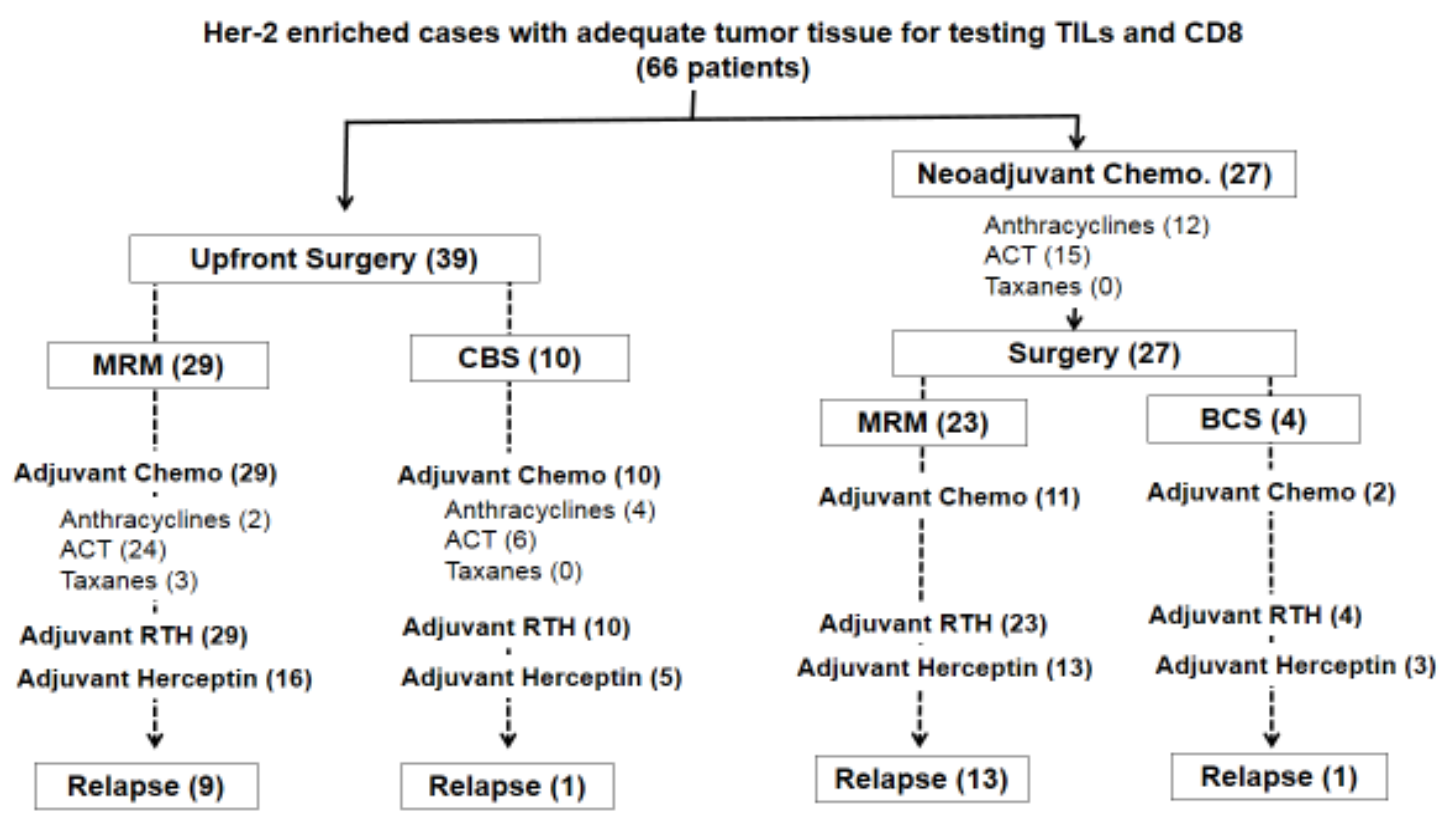

Figure 1. Flow Chart that Shows Treatment Received and Relapses in the 66 Her-2 Enriched Breast Cancer Patients in the Study. CBS, conservative breast surgery; MRM, modified radical mastectomy; RTH, radiotherapy

After assessment and classifying TILs levels into three groups in relapsed and non-relapsed patients, no statistically significance difference were found between the two groups $(p=0.321)$. Assessment of the level of TILs was done in the 23 relapsed cases and we found a statistically significant difference $(p=0.035)$ between those who received neoadjuvant chemotherapy and those who were managed with upfront surgery, where 11 patients with TILs less than $20 \%$ relapsed compared to 3 patients who were subjected to upfront surgery as shown in figure 3. Values for CD8 show as mean of $12 \%$ (range $0-50 \%$ ), median of $5 \%$ and mode of $1 \%$. CD8 antibody was analyzed as being present $(\geq 1 \%)$ or absent. Out of the 66 cases, $83 \%(n=20)$ showed absent $C D 8+$ cells and $17 \%$ $(n=4)$ were $C D 8+$ present. Relapses in CD8-ve cases were
$26 \%(n=6)$ and $74 \%$ in CD8+ve cases $(n=17)$. The mean and median DFS was 33.8 and 36 months respectively.

\section{Correlation between TILs/CD8 and study parameters}

Univariate analysis was done to identify possible correlation between TILs and other clinico-pathological parameters. There was a significant correlation between TILs and stage $(\mathrm{p}=0.028)$. Other factors failed to show statistically significant relationship.

CD8 analysis also showed a positive correlation with stage $(p=0.026)$ but also failed to show a statistically significant correlation with other parameters.

\section{Correlation between TILs and DFS}

TILs showed a significant positive correlation with

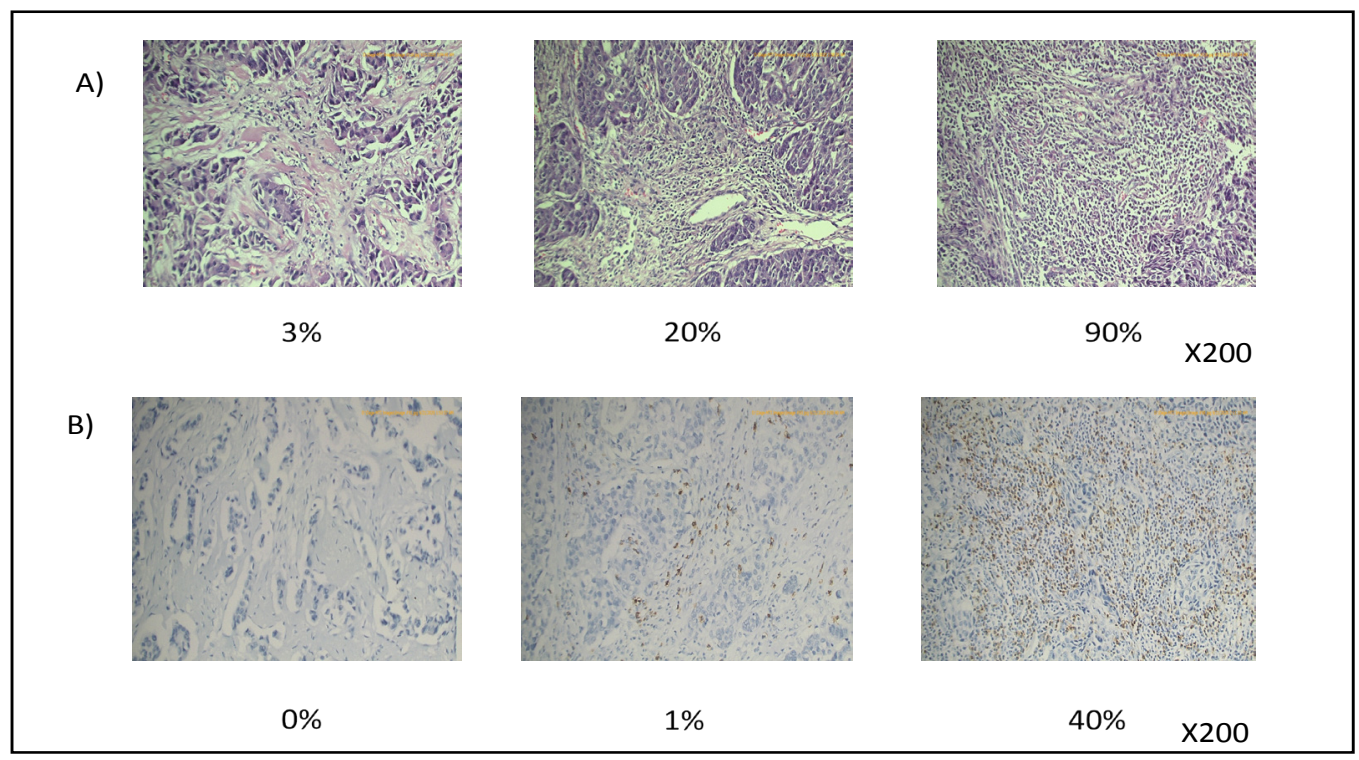

Figure 2. Representative Immunohistochemistry Images of A, TILs using H and E stain; B, CD8 using DAB chromogen. 
A)

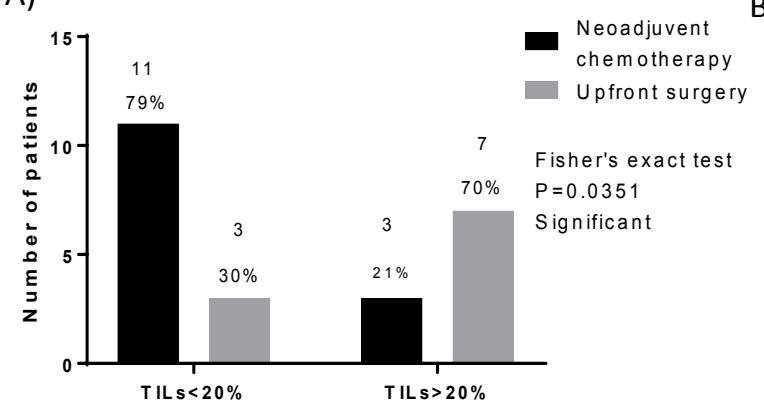

B)

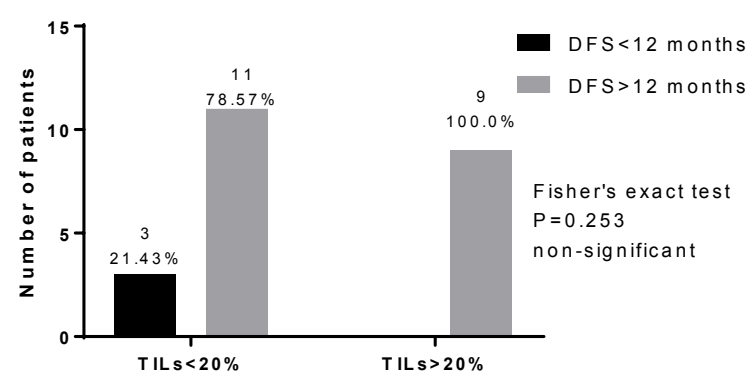

Figure 3. Level of Tumor TILs in the 23 Relapsed Patients According to A, the treatment received initially; B, disease free survival.

disease free survival $(\mathrm{p}=0.006)(95 \% \mathrm{CI}=0.099-0.532)$. The mean DFS was 23, 39, and 41 months for absent, intermediate and extensive TILs groups respectively; which was statistically significant $(\mathrm{p}<0.05)$. This shows that there is a linear correlation between level of TILs and DFS. Using Kaplan-Meier curve for analyzing correlation between TILs and DFS was not of statistical significance $(p=0.150)$ as shown in Figure 4.

Analysis of each level of TILs showed statistical significance in absent and intermediate groups, and not with extensive group. Other parameters in this study that may have affected the results were analyzed. Due
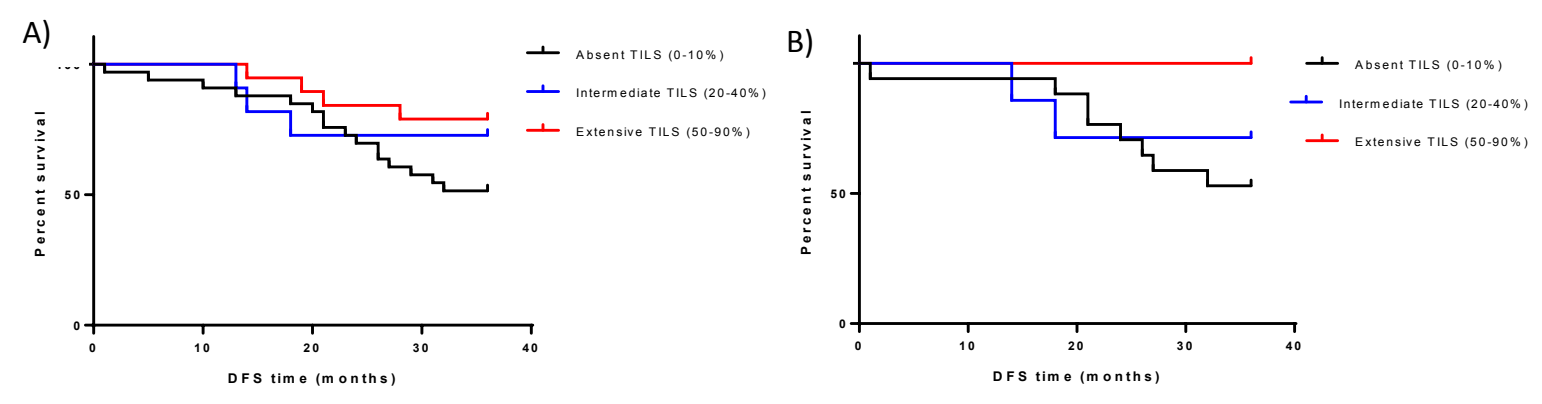

C)
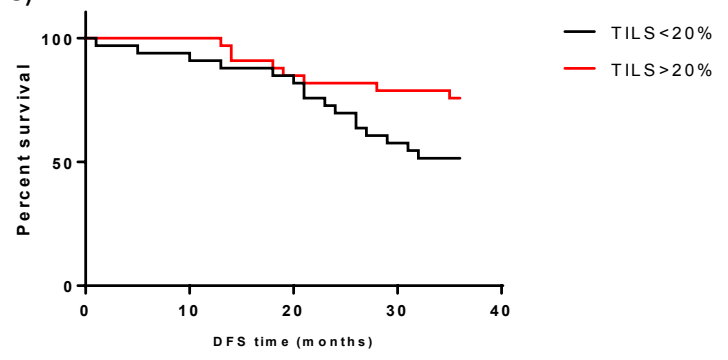

E)

D)

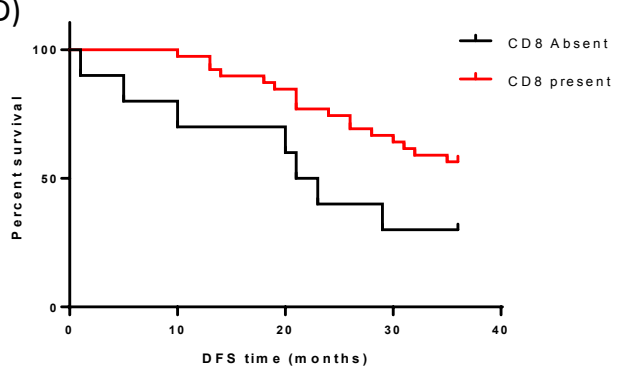

F)
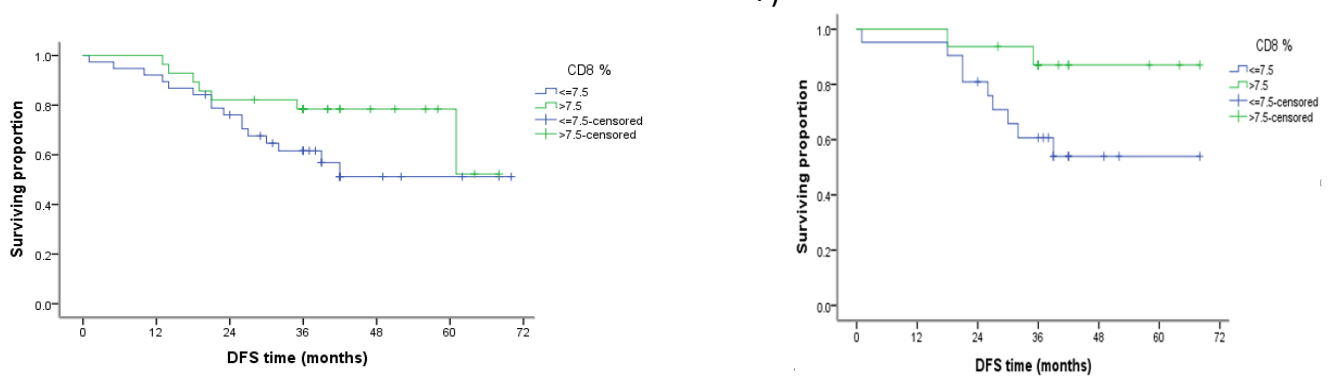

Figure 4. Kaplan-Meier Curves for Disease-free Survival According to A, level of TILS in total number of patients; B, level of TILS in patients who received trastuzumab in the adjuvant setting; $C$, level of intermediate and extensive TILS combined, D, percentage of CD8+ T-cells; E, CD8 in groups of $\leq$ and $>7.5 \%$ in total population; F, CD8 in groups of $\leq$ and $>7.5 \%$ in trastuzumab subgroup. 


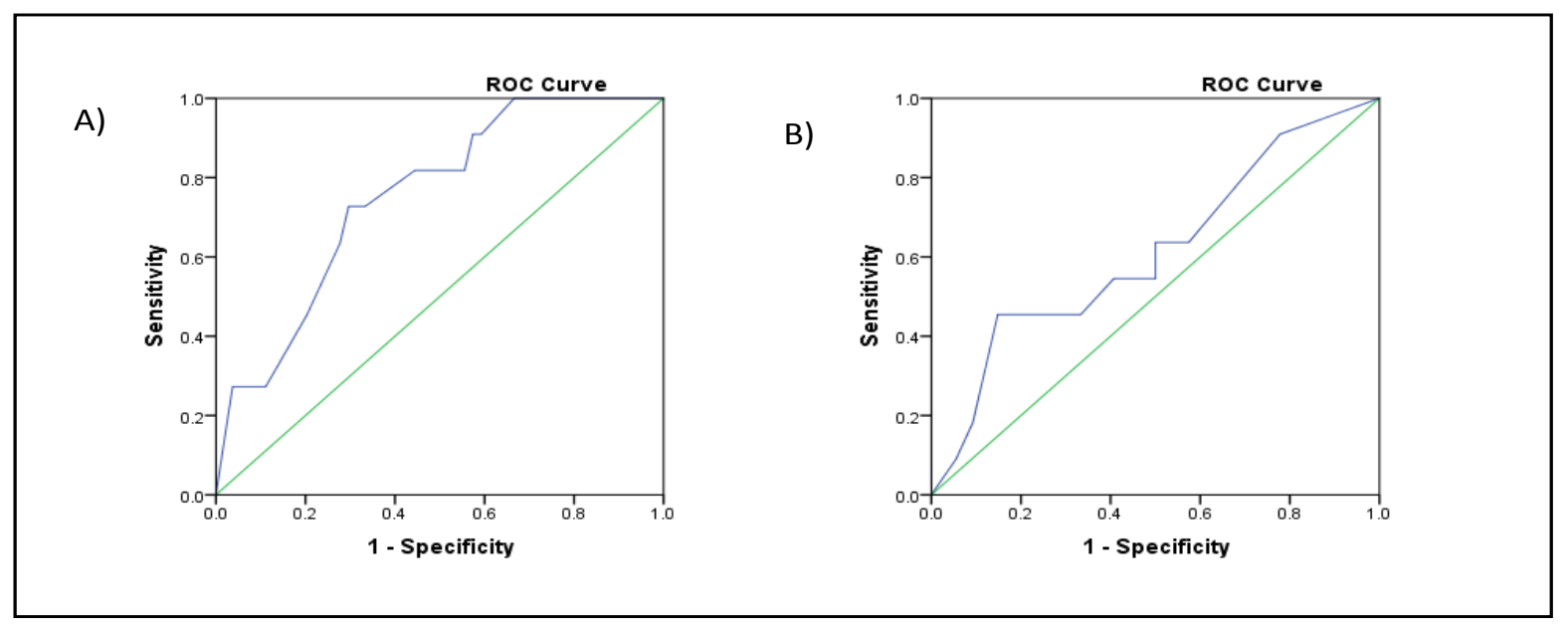

Figure 5. Receiver Operating Characteristic (ROC) Curve of A, TILs and grade; B, CD8 and stage.

to many regulations, trastuzumab was not offered to $44 \%$ of patients in this study which was a violation to NCI guidelines and standard of care. After omitting this subgroup, the correlation between DFS and intermediate / extensive groups were of statistical significance $(\mathrm{p}=0.0056 / 0.011$ respectively) and was not of statistical significance in absent TILs group $(\mathrm{p}=0.438)$. This relationship was reflected on Kaplan-Meier curve which showed significant clinical correlation between TILs and DFS in patients receiving trastuzumab $(\mathrm{p}=0.032)$. Correlation of intermediate and extensive TILs groups -combined- also showed a highly significant positive correlation with survival $(\mathrm{p}<0.0001)$.

Correlation between CD8+ lymphocyte subset and DFS

As for CD8+ analysis, the cut-off value of $\geq 1 \%$ was used initially and results showed a significant correlation with DFS among the same study population $(\mathrm{p}=0.028$, $95 \% \mathrm{CI}=0.02-0.48)$. CD8 marker was also shown to be highly representative of total TILs $\%$ ( $p<0.0001 ; 95 \%$ $\mathrm{CI}=0.766-0.906$ ), which suggests it may be used as a sole representative for TILs. Unlike TILs measurement, there is no standard cutoff value for defining CD8 positivity up to date. Besides the one percent positivity, two other methods were analyzed in order to find the value of highest significance - the ROC curve and mean CD8 level.

Defining a cut-off point for TILs using receiver operating characteristic (ROC) curve showed that level of $\geq 20 \%$ has the highest sensitivity of $82 \%$ and specificity of $56 \%$ ( $\mathrm{p}=0.009 ; 95 \% \mathrm{CI}=0.612-0.894)$. This suggests that values of intermediate and extensive TILs are positively correlated with increased DFS. The cut-off for CD8 was $7.5 \%$, with highest sensitivity of $52 \%$ and specificity of $64 \%(\mathrm{p}=0.008$; $\mathrm{CI}=0.557-0.827)$ (fig.5). Mean and median CD8 below the cut-off point ( $\leq 7.5 \%)$ were 3.3 and 1 respectively with standard deviation (SD) of 4.4. While mean and median CD8 $>7.5$ were 32 and 30 respectively with SD of 11.5 . Using the new cut-off points as a reference, mean DFS showed a significant difference between negative and positive $\mathrm{CD} 8$ subgroups after excluding the subset of patients who did not receive trastuzumab $(\mathrm{p}=0.055)$ (Figure 4). Using the mean CD8 level as a cutoff showed that values of $\geq 12 \%$ were significantly correlated with DFS. Patients with CD8 level of $<12 \%$ had a mean DFS of 31.3 months while those with values $\geq 12 \%$ had a mean DFS of 42.7 months ( $p=0.035$ ) $(95 \% \mathrm{CI}=0.863-22.35)$.

\section{Correlation between other prognostic factors and DFS}

Correlation between clinico-pathological parameters and DFS was of no statistical significance. These parameters include age, menopausal status, sidedness, body mass index, pregnancy and/or lactation, and family history. Results were also similar for pathological characteristics including lymphovascular invasion and presence of intraduct component. Patients with clinical stage IIB and IIIA seem to be highly correlated with survival; of which, tumor size shows highest significance $(\mathrm{p}<0.001,95.0 \% \mathrm{CI}=1.719-9.32)$.

\section{Discussion}

HER2 gene amplification or protein overexpression represents about $20-25 \%$ of breast cancers. This group is characterized by a more aggressive behavior and poor prognosis. The addition of the monoclonal antibody trastuzumab to the adjuvant treatment of HER2-positive breast cancer has shown a survival advantage in localized and metastatic disease. However, primary and secondary resistances to anti-HER 2 targets is observed in about $50 \%$ of HER2-postive patients which may be attributed to heterogeneity of cancer cells from genetic and non-genetic sources (Heppner et al., 2016).

In the current study, patients presented with median age of 50 years (range 28-71) with $6 \%$ were $\geq 65$ years of age. More than half of them were premenopausal. The lower median age in the current study is similar with Western data showing that $44 \%$ of HER 2 enriched cases presenting between 50 and 64 years of age which indicates that such aggressive breast cancer subtype has higher incidence among younger women (Howlader et al., 2014).

As observed in our study, not all of the patients received neoadjuvant chemotherapy despite that more than $80 \%$ of patients had tumors $>4 \mathrm{~cm}$ in maximum dimension and the majority had clinically palpable axillary lymph nodes, only $41 \%$ received neoadjuvant 
chemotherapy (NACT) which is considered the current standard of care. Reasons for low adoption of NACT were mainly patient or surgeon preference, which is no longer allowed at our institute due to compulsory incorporation of multi-disciplinary meetings in decision making for each patient. This has greatly reduced the incidence of suboptimal treatment for such patients. Neoadjuvant chemotherapy was anthracycline based $+/$ - taxanes as some of these patients were subjected to surgery after adequate clinical response and continued chemotherapy in the adjuvant setting. The combination of the higher stage at presentation and paucity of adoption of neoadjuvant therapy was reflected on the surgical decision favoring MRM as the curative surgery rather than CBS ( 79 vs. $21 \%$, respectively), in addition that wire localization procedure was not accessible for many patients. This contrasts with the situation in Western countries where the earlier presentation and the use of neoadjuvant therapies lead to more adoption of CBS which is associated with better quality of life and is preferred by most patients compared with mastectomy (De Haes et al., 2003).

It was observed that more relapses were encountered in patients who received neoadjuvant chemotherapy compared with those who were subjected to upfront surgery. This may be explained that a large subset of patients received neoadjuvant anthracyclines only without taxanes; also, patients were preferentially selected for upfront surgery who had relatively "less" locally advanced disease; in addition to the considerable number of patients who did not receive trastuzumab. The combination of these factors may explain the dismal outcome for this group of patients in the study. Now, cases are discussed in multidisciplinary meetings, therefore better decisions are made.

Not all patients received trastuzumab during the course of therapy. Although it has been standardized in Egypt since more than 10 years, many patients did not have access to the drug at that period and there reasons are diverse; these include age policy, insurance issues and transient periods of drug shortage.

Only quarter of the patients who received neoadjuvant chemotherapy achieved pathological complete response $(26 \%)$ which is much lower than the standard rate, according to a meta-analysis of 52 studies that showed a $36.4 \%$ pCR for HER2 +ve patients (Spring et al., 2020). This can be explained by the fact that not all chemotherapy course was given in neoadjuvant setting and was partly completed after surgery.

Despite the extensively investigated association between the tumor infiltrating lymphocytes and prognosis in breast cancer, the effect of the lymphocyte subpopulation ratios still remains unclear (Ravelli et al., 2017). High levels of TILs indicate better prognosis than low TIL levels in HER2+ breast cancer patients. Raphael et al. (Raphael et al., 2016) reported that the higher the histological cancer grade the higher was the percentage of TILs. Our study did not show a similar correlation, probably because the majority of the patients had grade 2 tumors, therefore comparison between variables was not statistically representative.

Over the past years, in several studies, TILs have been shown to be a positively correlated with survival in breast cancer especially in TNBC and HER 2 enriched disease (Jin and $\mathrm{Hu}, 2020$ ). Our results echoed these findings with linear correlation with level of TILs and DFS. To our knowledge, a cut-off value of TILs level was not focused upon in literature, yet, values $>20$ represent intermediate and extensive TILs groups according to international TILs Working Group and therefore the cut-off value reflects the standard guide of TILs.

CD8+ Cytotoxic T cells in particular has been focused upon in tumor microenvironment for several reasons. They have demonstrated reactivity to tumor-derived self-epitopes in addition to being a potent cytotoxic agent. They are also the most prevalent subset of lymphocyte in tumor microenvironment and poses a positive prognostic effect in breast cancer as well as other tumor types (Ziai et al., 2018).

Seventy-five percent of TILs are found to be T cells. Among these, CD8+ T cells represent the class of lymphocytes that correlate better with overall favorable clinical outcomes, usually infiltrating breast lesions in the largest proportion (Ahn et al., 2015). CD8+ T cells represented $80 \%$ of our studied specimens. It has been widely documented that the presence of high rates of infiltrating CD8 $+\mathrm{T}$ cells is associated with overall longer survival rates (Mahmoud et al., 2011). These findings are comparable to our study, in which relapses were much higher in CD8 negative subgroup compared to positive counterpart (18 versus 5 patients for negative and positive CD8 respectively). In a recent study, CD8+ lymphocytes cells were significantly associated with disease free survival in young breast cancer patients under the age 40 , but not in their older counterparts (Jin and $\mathrm{Hu}, 2020$ ). In another study, TILs grade of the primary tumor was not a significant prognostic factor. However, the prognosis of the cases with high CD8+ TILs density was significantly better than those with low CD8-positive TILs density (Kurozumi et al., 2019).

CD8 antibody testing was assessed in tumor infiltrating lymphocytes. Counting of CD8+ve T lymphocytes was done in tumor-associated stromal and/or perivascular spaces with high-power fields (HPF) at $\times 400$ magnification. Five areas were randomly selected and the average was calculated. This technique was largely similar to that used in other studies. In a recent study, CD8+ and FOXP3+ cells were counted within the tumor and the surrounding area (within $1 \mathrm{~mm}$ from tumor-invading front). The average absolute count of positive staining cells in $10 \mathrm{HPF}$ was used instead of 5 (Zhao et al., 2020).

The value of $1 \%$ positivity was considered for CD8. This value was utilized in our studies in comparing patient groups. Although a significant relationship was found between CD8+ levels of $\geq 1 \%$ and survival, other cut-offs were used to highlight the value of greatest significance and minimize the risk of false positive results. In the study conducted by (Ziai et al., 2018), several cut-offs for CD8+ lymphocytes were considered including $1 \%, 2 \%$, $5 \%$ and $10 \%$. These values were selected based on mean CD8 value, sensitivity and specificity. He concluded that the mean value for CD8+ cells in a tumor sample gives highest accuracy. In our study, the ROC value of 7.5\%

Asian Pacific Journal of Cancer Prevention, Vol 23 
showed marginal significance with DFS in trastuzumab subgroup only, while the mean value of $12 \%$ showed highest significance compared to other cut-offs that were used. This suggests that higher values of CD8 are better correlated with survival, however a definitive cut off is yet to be determined.

Despite the limitations associated with the number of tumor samples included in this analysis, our results highlight the practical importance of TILs and specifically CD8+ lymphocytes as a prognostic value and may suggest a predictive utility for different treatment regimens.

In conclusion, in the current study, we concluded that there is a direct correlation between level of TILs and survival in HER2 enriched breast cancer patients. Intermediate and extensive levels for TILs have shown to be highly correlated with DFS in such population provided that patients receive adequate anti HER2 therapy. CD8 lymphocytes may act as a surrogate marker for TILs. Level of $12 \%$ was shown to have the highest clinical significance as regards DFS in this study group.

\section{Author Contribution Statement}

Mostafa M Saber: Writing-Original draft preparation, Investigation. Heba M. El Zawahry: Supervision, Project administration. Amany M. Hilal: Conceptulization, Methodology. Amany A. Abou-Bakr: Data Curation, Resources. Alfred E. Namour: Validation. Mona M Saber: Formal analysis, Writing-Review and Editing.

\section{Acknowledgements}

This work was supported and in part by the Medical oncology and Pathology Departments, National Cancer Institute, Cairo University. The study protocol was approved by the Institutional Review Board (IRB) of the national cancer institute $(\mathrm{NCI})$. This research received no funding from any research agency.

\section{Conflict of interest}

The authors declare that there is no conflict of interest.

\section{References}

Adams S, Gray RJ, Demaria S, et al (2014). Prognostic value of tumor-infiltrating lymphocytes in triple-negative breast cancers from two phase III randomized adjuvant breast cancer trials: ECOG 2197 and ECOG 1199. J Clin Oncol, 32, 2959-66.

Ahn SG, Jeong J, Hong SW, Jung WH (2015). Current issues and clinical evidence in tumor-infiltrating lymphocytes in breast cancer. $J$ Pathol Transl Med, 49, 355-63.

Denkert C, Loibl S, Noske A, et al (2010). Tumor-associated lymphocytes as an independent predictor of response to neoadjuvant chemotherapy in breast cancer. J Clin Oncol, 28, 105-13.

ELSTON CW, ELLIS IO (1991). pathological prognostic factors in breast cancer. I. The value of histological grade in breast cancer: experience from a large study with long-term followup. Histopathology, 19, 403-10.

De Haes JCJM, Curran D, Aaronson NK, Fentiman IS (2003). Quality of life in breast cancer patients aged over 70 years, participating in the EORTC 10850 randomised clinical trial.
Eur J Cancer, 39, 945-51.

Heppner BI, Untch M, Denkert C, et al (2016). Tumor-infiltrating lymphocytes: A predictive and prognostic biomarker in neoadjuvant-treated HER2-positive breast cancer. Clin Cancer Res, 22, 5747-54.

Howlader N, Altekruse SF, Li CI, et al (2014). US incidence of breast cancer subtypes defined by joint hormone receptor and HER2 status. J Nat Cancer Instit, 106.

Jin YW, Hu P (2020). Tumor-infiltrating cd $8 \mathrm{t}$ cells predict clinical breast cancer outcomes in young women. Cancers, 12.

Kurozumi S, Inoue K, Matsumoto H, et al (2019). Prognostic utility of tumor-infiltrating lymphocytes in residual tumor after neoadjuvant chemotherapy with trastuzumab for HER2positive breast cancer. Sci Rep, 9, 1-8.

Liu S, Lachapelle J, Leung S, et al (2012). CD8 +lymphocyte infiltration is an independent favorable prognostic indicator in basal-like breast cancer. Breast Cancer Res, 14.

Mahmoud SMA, Paish EC, Powe DG, et al (2011). Tumor-infiltrating CD8+ lymphocytes predict clinical outcome in breast cancer. J Clin Oncol, 29, 1949-55.

Raphael J, Gong IY, Nofech-Mozes S, et al (2016). Tumour infiltrating lymphocytes and stromal CD68 in early stage HER2 positive breast cancer. $J$ Clin Pathol, 69, 552-5.

Ravelli A, Roviello G, Cretella D, et al (2017). Tumor-infiltrating lymphocytes and breast cancer: Beyond the prognostic and predictive utility. Tumor Biol, 39.

Salgado R, Denkert C, Demaria S, et al (2015). The evaluation of tumor-infiltrating lymphocytes (TILS) in breast cancer: Recommendations by an International TILS Working Group 2014. Ann Oncol, 26, 259-71.

Seo AN, Lee HJ, Kim EJ, et al (2013). Tumour-infiltrating CD8+ lymphocytes as an independent predictive factor for pathological complete response to primary systemic therapy in breast cancer (2013). Br J Cancer, 109, 2705-13.

Spring LM, Fell G, Arfe A, et al (2020). Pathologic complete response after neoadjuvant chemotherapy and impact on breast cancer recurrence and survival: A Comprehensive Meta-analysis (2020). Clin Cancer Res, 26, 2838-48.

Symmans WF, Peintinger F, Hatzis C, et al (2007). Measurement of residual breast cancer burden to predict survival after neoadjuvant chemotherapy. J Clin Oncol, 25, 4414-22.

Tan PH, Ellis I, Allison K, et al (2020). The 2019 World Health Organization classification of tumours of the breast. Histopathology, 77, 181-5.

Zhao J, Meisel J, Guo Y, et al (2020). Evaluation of PD-L1, tumor-infiltrating lymphocytes, and CD8+ and FOXP3+ immune cells in HER2-positive breast cancer treated with neoadjuvant therapies. Breast Cancer Rese Treat, 183, 599-606.

Ziai J, Gilbert HN, Foreman O, et al (2018). CD8+ T cell infiltration in breast and colon cancer: A histologic and statistical analysis. PLoS One, 13.

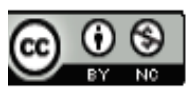

This work is licensed under a Creative Commons AttributionNon Commercial 4.0 International License. 